Deborah A. Payne*, Katarina Baluchova, Graciela Russomando, Parviz Ahmad-Nejad, Cyril Mamotte, Francois Rousseau, Ron H.N. van Schaik, Kristin Marriott, Masato Maekawa and K.C. Allen Chan, on behalf of the IFCC Committee on Molecular Diagnostics

\title{
Toward harmonization of clinical molecular diagnostic reports: findings of an international survey
}

https://doi.org/10.1515/cclm-2017-1080

Received November 21, 2017; accepted March 6, 2018; previously published online May 5, 2018

\section{Abstract}

Background: The International Organization for Standardization (ISO) 15189 standard provides recommendations for the postexamination reporting phase to enhance quality in clinical laboratories. The purpose of this study was to encourage a broad discussion on current reporting practices for molecular diagnostic tests by conducting a global survey of such practices.

Methods: The International Federation of Clinical Chemistry and Laboratory Medicine's Committee for Molecular Diagnostics (IFCC C-MD) surveyed laboratories on selected ISO 15189 recommendations and topics. The survey addressed the following aspects: (1)

\footnotetext{
* Corresponding author: Deborah A. Payne, American Pathology Partners-Unipath LLC - Clinical Trials and Development, Denver, CO, USA

Katarina Baluchova: Comenius University in Bratislava, Jessenius Faculty of Medicine in Martin, Biomedical Center, Division of Oncology, Martin, Slovakia

Graciela Russomando: Universidad Nacional de Asunción, Molecular Biology and Biotechnology Department, Instituto de Investigaciones en Ciencias de la Salud, San Lorenzo, Paraguay

Parviz Ahmad-Nejad: Institute for Medical Laboratory Diagnostics, Centre for Clinical and Translational Research (CCTR), Wuppertal, Germany; and Universitat Witten/Herdecke, Witten, Germany Cyril Mamotte: Curtin University, School of Biomedical Sciences and CHIRI Biosciences, Perth, Australia

Francois Rousseau: CHU de Québec, Université Laval, Department of Laboratory Medicine and Department of Molecular Biology, Medical Biochemistry and Pathology, Faculty of Medicine, Centre de recherché, Québec City, Quebec, Canada

Ron H.N. van Schaik: Erasmus Medical Center, IFCC Expert Center Pharmacogenetics, Department of Clinical Chemistry, Rotterdam, The Netherlands

Kristin Marriott: KSchmidt Consulting, LLC., Littleton, CO, USA Masato Maekawa: Hamamatsu University School of Medicine, Department of Laboratory Medicine, Hamamatsu, Japan K.C. Allen Chan: The Chinese University of Hong Kong, Department of Chemical Pathology, Hong Kong SAR, P.R. China
}

laboratory demographics, (2) report format, (3) result reporting/layout, (4) comments in report and (5) interpretation and clinical decision-making information. Additionally, participants indicated categories needing standardization.

Results: Sixteen responses from laboratories located in Asia, Europe, the Middle East, North America and South America were received. Several categories yielded 100\% agreement between laboratories, whereas other categories had less than or equal to 50\% concordance. Participants scored "nomenclature" and "description of methodologies" as the two most frequently cited aspects needing standardization.

Conclusions: The postexamination phase requires extensive and consistent communication between the laboratory, the healthcare provider and the end user. Surveyed laboratories were most likely to follow explicit ISO 15189 recommendations vs. recommendations when the term(s) "where appropriate or where applicable” was used. Interpretation and reporting of critical values varied among participants. Although the outcome of this study may not fully represent the practices of all molecular testing laboratories in countries around the world, the survey identified and specified several recommendations that are requirements for harmonized reporting in molecular diagnostics.

Keywords: clinical genomics; infectious diseases; inherited diseases; ISO 15189; molecular pathology; noninvasive prenatal testing; oncology; pharmacogenomics; precision medicine diagnostics; transplantation.

\section{Introduction}

Molecular diagnostic test results facilitate patient management by providing the healthcare provider with actionable information. Improving patient care requires implementation of quality management systems and standardized technical practices by the medical laboratory. These practices focus mainly on preexamination, examination and postexamination processes (formerly referred to as preanalytic, 
analytic and postanalytic processes) [1]. Although many guidance documents are available, the International Organization for Standardization (ISO) 15189 standard (2012) was most frequently cited by clinical laboratories in the previous survey conducted by the International Federation of Clinical Chemistry and Laboratory Medicine Committee for Molecular Diagnostics (IFCC C-MD) [1-6]. In order to educate and support standardization for molecular diagnostic testing, the IFCC-MD previously published a case-based review that addressed preexamination factors that could affect molecular diagnostic assay results [2]. As a follow-up to this effort, the IFCC C-MD conducted a survey of laboratories performing molecular diagnostic tests to determine reporting practices. This study provided baseline data on current reporting practices used by molecular diagnostic laboratories. In addition, data from the study identified several aspects of reporting that could be improved through standardization. The findings from this study aim to encourage a broad discussion on current reporting practices in molecular diagnostics.

\section{Survey methodology}

A survey was designed to capture data on reporting practices for molecular diagnostics through a collaborative process with an international molecular diagnostic expert panel. Questions were derived from the ISO 15189 standard section (specifically sections 5.8 and 5.9 Reporting of Results and Release of Results) and by the expert panel. Some questions are not required by the ISO standard and may not currently be part of best practice guidelines nor were formally recommended by Health-TechnologyAssessment (HTA) bodies. These additional questions were deemed important to the IFCC C-MD because they provided insight into the trends and novel aspects associated with reporting molecular diagnostic results. The survey for molecular reporting practices addressed the following sections: (1) laboratory demographics, (2) report format, (3) result reporting/layout, (4) comments in report and (5) interpretation and clinical decision making information. Additionally, participants were asked to designate areas of molecular reporting that need standardization. Surveyed laboratories included all members of the IFCC C-MD, the IFCC network of molecular centers and molecular diagnostic laboratories identified by committee members [7, 8]. IFCC C-MD members were nominated by regional clinical chemistry organizations to represent molecular diagnostics. Laboratories seeking to be designated an IFCC Network of Molecular Diagnostic Centers must obtain approval by the IFCC C-MD [9]. The survey consisted of 69 questions and had both multiple choice and open answer formats (Supplementary Material). The survey took approximately 45 minutes to complete The data were collected using Surveymonkey.com using an link embedded in an e-mail [10]. Over 30 laboratories were invited by e-mail to participate. All participants were asked as part of the survey to identify the specific area(s) of molecular diagnostics as part of the inclusion criteria of the survey.

\section{Results}

\section{Survey participant demographics}

In order to ascertain laboratory demographics, several questions addressed accreditation, guidance documents, country, specialization, etc. Figure 1 illustrates the molecular subspecialties reported by the survey participants. Some laboratories reported more than one area of molecular diagnostics. Inherited disease testing was performed by $75 \%$ of respondents followed by personalized medicine/precision diagnostics, oncology, infectious disease testing, pharmacogenomics, non-invasive prenatal testing and transplantation. One laboratory responding as "other" stated that they performed testing of in vitro diagnostic kits on behalf of their national ministry of health. The second laboratory responding as "other" stated plans to implement oncology and precision diagnostics in the near future. The regions represented in the survey included Europe (France, Germany and Slovakia), North America (Canada, Mexico and United States of America) and South America (Paraguay), Asia (China-Hong Kong, India and Japan) and the Middle East (Iran and Israel). Some laboratories were located in regions that did not require accreditation by governmental or provincial regulatory entities. Not all laboratories were required by their health jurisdiction to be accredited by a governmental agency, but all laboratories reported using standards/guidelines to insure patient care for molecular testing. Standards, accrediting/certifying organizations and guidance documents are listed in Tables 1 and 2, respectively. Additional resources identified by the IFCC C-MD were added following review of the data for these tables.

\section{Report format}

\section{Laboratory and patient demographics}

The laboratory can define the format in which it chooses to report patient results. Laboratories use the following 


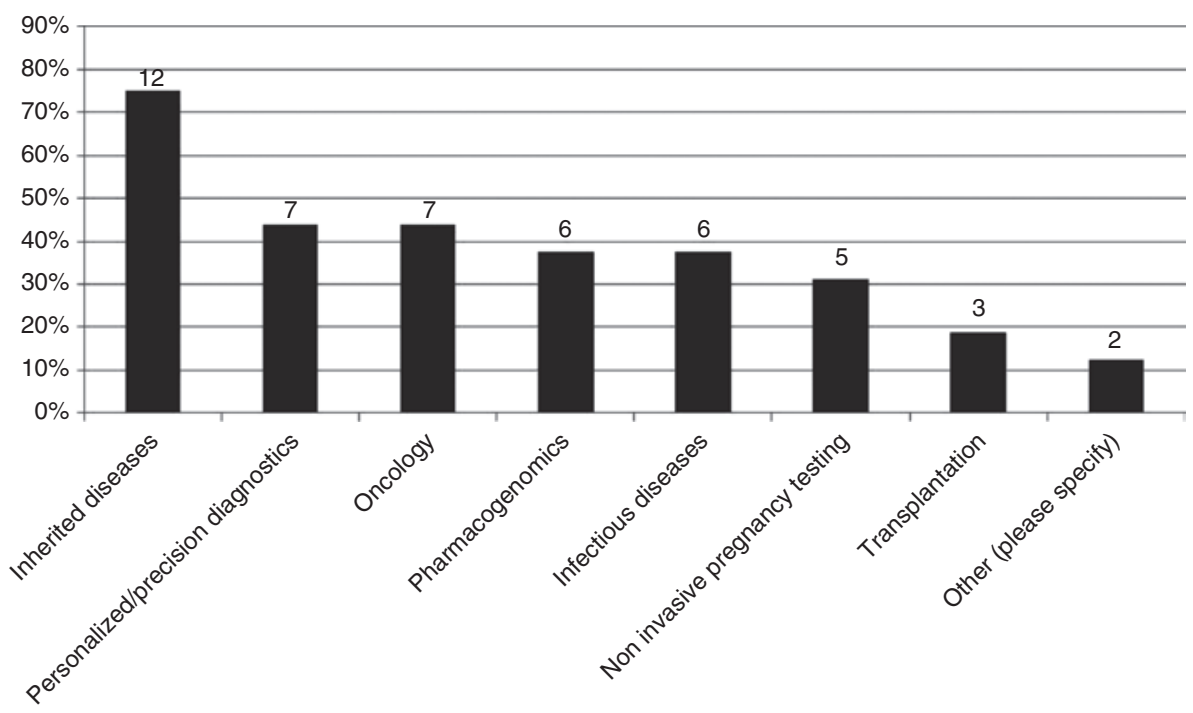

Figure 1: Subspecialties of molecular diagnostic laboratories participating in the survey.

The number of laboratories reporting specific areas of specialization is listed above each category.

Table 1: Considered standards, accreditation and certifications bodies.

College of American Pathologists (CAP) [11]

Clinical Laboratory Improvement Amendments (CLIA) [12, 13]

Comité Français d'Accréditation (COFRAC) [14]

Deutsche Akkreditierungsstelle (DAkkS) [15]

European Accreditation (EA) ${ }^{\mathrm{a}}$ [16]

Health Reference Laboratory, Ministry of Health of IRAN [17]

International Accreditation Foruma (IAF) [18]

International Laboratory Accreditation Cooperation ${ }^{a}$ (ILAC) [19]

International Organization of Standardization (ISO) [20]

National Accreditation Board of Testing and Calibration Laboratories

(NABL) [21]

National Pathology Accreditation Advisory Council (NPAAC) via

National Association of Testing Authorities, Australia (NATA) [22]

Netherlands Institute for Accreditation ${ }^{a}$ (NIAZ) [23]

Slovak National Accreditation Service (SNAS) [24]

${ }^{\mathrm{a}}$ Organizations were not reported in the survey but were added by authors for completeness.

mechanisms to release data: paper/faxed (also known as "hard" copies) copies, electronic reports to the healthcare provider, electronic reports to electronic medical record (EMR), web-based reporting and reports directly to patient. Some laboratories use a combination of the above.

The format of the report should provide identifying information regarding both the patient and the laboratory performing the test. All survey participants stated that the identity of the laboratory, location and address was included in the molecular diagnostic report. This information provides the healthcare provider with the ability
Table 2: Considered organizations providing standards and guidance for molecular diagnostic laboratories.

American College of Medical Genetics (ACMG) [25]

Association of Clinical Genetic Science (ACGS) ${ }^{a}$ [26]

Association for Molecular Pathology (AMP)a [27]

Australian Government Department of Health Therapeutic Goods

Administration [28]

Canadian Standards Associations (CSA) [29]

College of American Pathologists (CAP) [11]

Comité Français d'Accréditation (COFRAC) [14]

Clinical Laboratory Standardization Institute (CLSI) [30]

European Society for Human Genetics (ESHG) ${ }^{\mathrm{a}}$ [31]

Japanese Committee for Clinical Laboratory Standards (JCCLS) [32]

International Organization of Standardization (ISO) [20]

International Federation for Clinical Chemistry and Laboratory

Medicine (IFCC) [7]

Israel Health Ministry Standardization [33]

National Accreditation Board of Testing and Calibration Laboratories (NABL) [21]

National Pathology Accreditation Advisory Council (NPAAC) [22]

The Organisation for Economic Co-operation and Development

$(\mathrm{OECD})^{\mathrm{a}}$ [34]

Richtlinie der Bundesärztekammer; German Chamber of

Physicians [35]

Swiss Society of Medical Genetics (SGMG)a [36]

${ }^{a}$ Organizations were not reported in the survey but were added by authors for completeness.

to contact the laboratory if necessary. Report design may repeat selected information on every page of the report. For example, ISO 15189 recommends that the patient name and the location of the patient be included on every page. The patient location may be interpreted as that of a clinic or inpatient facility. All surveyed laboratories 
Table 3: Statistics on surveyed laboratories responding to selected ISO 15189 recommendations.

\begin{tabular}{|c|c|c|c|c|}
\hline Section & Selected ISO 15189 recommendations $^{\mathrm{a}}$ & Yes & $\begin{array}{r}\text { No. of } \\
\text { responders }\end{array}$ & Percentage \\
\hline & The laboratory report should include: & & & \\
\hline 5.8.2.a & Comment for sample quality that might compromise examination results & 13 & 15 & 87 \\
\hline 5.8.3.b & The identification of the laboratory, location and address ${ }^{b}$ & 16 & 16 & 100 \\
\hline 5.8.3.d & Patient identification on each page of the report & 16 & 16 & 100 \\
\hline 5.8.3.d & Patient location on each page of the report & 14 & 16 & 88 \\
\hline 5.8.3.e & Name or unique identifier of the requester and the requester's contact details & 13 & 16 & 81 \\
\hline 5.8.3.f & Date of the primary sample collection & 14 & 16 & 88 \\
\hline 5.8.3.g & Type of primary specimen & 14 & 16 & 88 \\
\hline 5.8.3.0 & Date of the report & 16 & 16 & 100 \\
\hline 5.8.3.0 & Time of release (if not contained in the report, readily available when needed) & 13 & 16 & 81 \\
\hline 5.8.3.p & Page numbers to total page number (for example, page 1 of 5) & 13 & 15 & 87 \\
\hline 5.8.3.m & $\begin{array}{l}\text { Identification of examinations undertaken as part of a research or development program } \\
\text { and for which no specific claims on measurement performance are available }\end{array}$ & 3 & 15 & 20 \\
\hline 5.8.3.n & Identification of the person(s) reviewing the results and authorizing release of report & 10 & 15 & 67 \\
\hline 5.8.3.n & $\begin{array}{l}\text { If identification of the person(s) reviewing the results and authorizing release of report is not } \\
\text { contained in the report, this information is readily available when needed }\end{array}$ & 7 & 7 & 100 \\
\hline 5.9. Note 1 & $\begin{array}{l}\text { For results of some examinations (e.g. certain genetic or infectious disease examinations), } \\
\text { special counseling may be needed. The laboratory should endeavor to see that results with } \\
\text { serious implications are not communicated directly to the patient without the opportunity for } \\
\text { adequate counseling }\end{array}$ & 8 & 14 & 57 \\
\hline
\end{tabular}

aCISO. This material is excerpted from ISO 15189:2012 with permission of the American National Standards Institute (ANSI) on behalf of ISO. Copies of the standard can be purchased from ANSI at https://webstore.ansi.org. All rights reserved. bISO only requires the identification of the laboratory.

reported that the patient name was included on every page of the report. Two respondents (13\%) stated that their molecular diagnostic report did not include the location of the patient on every page (Table 3). Similarly, ISO 15189 recommends including the name and contact information from the healthcare provider on the report. Compliance was $81 \%$ for this recommendation. Inclusion of the patient location (such as the clinic or outpatient facility) on all pages of the report ensures that each page can be delivered to the healthcare provider. For this reason, reports should be numbered and include the total number of pages contained in the report (such as page 1 of 2). Socalled "hard copies" or faxed copies of multipage reports are at risk of being incomplete if pages become separated from each other during the reporting process. Even blank pages generated during the process should be included in the report to assure that the complete report has been delivered to the healthcare provider that ordered the test(s). Eighty-seven percent of laboratories state the total number of pages in their report. The IFCC C-MD requested information inquiring whether laboratories included two unique patient identifiers in their reports. Reports including two unique identifiers (such as patient name and date of birth) improve the process that correct results are delivered to patients with the same or similar names, an aspect that is particularly important for studies on related individuals. Eighty-eight percent $(n=14)$ of laboratories included two unique identifiers in their report.

\section{Sample demographics}

Quality and interpretation of test results can be affected by auxiliary information such as date and time of specimen collection. This information enables the laboratory to identify potential preexamination issues that could impact reportable results or interpretation [2]. For instance, delays in receiving the specimen could render the specimen unacceptable. Date of collection also permits the healthcare provider to monitor test results that vary with treatment. Compliance with the date of collection was $88 \%$, whereas compliance with the time of collection was $75 \%$. Including the time of collection is an example of an ambiguous recommendation because the specific recommendation states "...time, when available and relevant to patient care". Including the time of collection does not universally impact the quality of a molecular result or its interpretation. However, the time of collection may be required for test interpretation if treatment is started before or after specimen collection. For instance, molecular levels of analytes such as tumor biomarkers associated circulating cell free DNA (cfDNA) or infectious agents may change 
following treatments such as chemotherapy, transfusion or antiviral/antibiotic administration. The date that the report was released was reported by $100 \%$, whereas laboratories who also included the time of the report was $81 \%$. The inclusion of the time of the report should be available to the provider if not included on the report according to ISO 15189. For a quality monitoring perspective, tracking the date and time of release of test results could be valuable metrics used to assess sample processing. Interpretation of laboratory results by the healthcare provider may be influenced by the specimen type, and for this reason, specimen type should be included in the report. For instance, a healthcare provider may request the same test on bone marrow and peripheral blood for molecular monitoring of hematologic disorders. Eighty-eight percent of laboratories included the specimen type on reports. Although not included in the ISO 15189 recommendations for reporting, the surveyed laboratories were asked if the specific collection media (or device) was included in the report. A lower number of laboratories (56\%) included the specimen collection media or device on the report. Molecular results and interpretation can be affected by the collection method, as exemplified by the unsuitability of heparinized blood samples in some analytical systems.

\section{International diagnostic codes}

Another question that was asked by the IFCC C-MD but not required by ISO 15189 inquired if laboratories included international diagnostic codes in the report. A minority of laboratories (38\%) used the World Health Organization (WHO) designated International Classification of Diseases [ICD-10] codes and/or Online Mendelian Inheritance in Man (OMIM $\left.{ }^{\circledR}\right)$ codes as references for the clinical indications/disease in the report $[37,38]$. For example, the ICD-10 code for BCR-ABL1 positive Chronic Myeloid Leukemia (CML) is C92.1, whereas the OMIM code is 608232. Clinical classifications in the report support interpretation of the molecular results. In some instances, testing performed on a specimen using a correct diagnostic code provides evidence of appropriate test utilization and can aid interpretation. In some locales, discrepancies between the diagnostic code and the test result may prevent reimbursement for the test.

\section{Molecular diagnostic test methodology}

Molecular methods differ in their analytic and clinical performance characteristics that can negatively impact commutability of results. Describing the molecular method in the report provides information, aids interpretation of test results and enables identification of samples which may require a retrospective reanalysis where methods are ultimately deemed to be unreliable (e.g. based on proficiency testing). The ISO 15189 recommendation for including the measurement/examination method in the report is ambiguous in that the phrase "where appropriate" accompanies the recommendation. Although this recommendation is open to interpretation, $94 \%(n=15)$ of laboratories identified the methodology in the report. Laboratories reporting the method in the body of the report were $88 \%$ $[n=14]$. Other locations for methods description included the disclaimer section or a link to an outside source. Although not a recommendation by ISO 15189, some agencies require that the laboratory declare if the method has been validated by a regulatory agency, which may be particularly relevant for laboratory developed tests. Laboratories (specifically 38\%) reported if the method was cleared or approved by a regulatory agency and included the statement either in the body, disclaimer and/or in a link outside of the report. A lower percentage of laboratories (31\%) disclosed if the laboratory-developed molecular tests were validated. Information on the method/examination provides the healthcare provider the opportunity to interpret changes in diagnostic values that may result from technical artifacts rather than changes caused by pathophysiology of the disorder.

\section{Report size and content}

Space restrictions on medical report can be an issue. No recommendations on specific font size, report length or reporting mechanism (human readable) are mentioned in ISO 15189. Some healthcare providers prefer short reports. In this survey, reports ranged from one to three pages with most laboratories utilizing a one page format (specifically, 47\%) compared to a three page format (specifically, 13\%). Laboratories may maximize the information on the report by altering font size or, as previously mentioned, using links to outside information resources such as websites (specifically disclaimers or statement regarding the measurement procedure). Laboratories can elect to emphasize information by varying font size. Sixty-three percent of laboratories used different size fonts, color fonts and/or underlined words to differentiate "detected" or "affected" from "not detected" or "not affected". By contrast, 67\% laboratories used the same font size as the rest of the text in the body to describe the measurement method. Of the laboratories that changed their font size, the font was reduced to size 


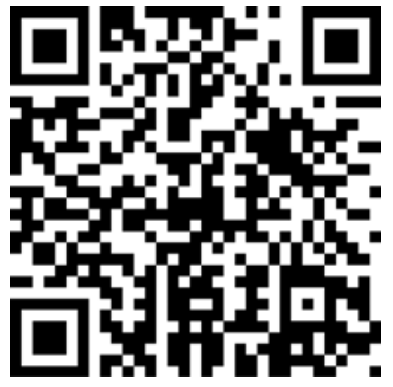

Figure 2: Non-human readable codes. Quick Response Code (QR) for the IFCC C-MD.

8 and 6. Thirty percent of laboratories include non-human readable code (for example, Quick response code [QR] or barcodes) on their reports (Figure 2). A balance between insuring adequate information for test interpretation and readability may be a challenge for laboratories seeking to maximize the content of the report while minimizing the number of pages for the report.

Laboratories in multilingual countries were surveyed to determine the number of languages used to report the result. Ninety-three percent of respondents representing 14 countries used a single language in the report. One country reported results in two languages (specifically Persian and English). Most countries report in the language spoken by the majority of the population. However, some countries used English as the reporting language even if the majority of the population spoke a different language. Reporting in English in these countries occurred prior to the country gaining their sovereign authority (such as when a region was occupied or colonized by an English speaking country, or if English was the language for medical education). ISO 15189 does not have a recommendation for selecting the primary language of the report.

\section{Reportable results}

\section{Nomenclature}

ISO 15189 recommends that results should be unambiguous and that standardized nomenclature and units be used in reporting results. Standardization of nomenclature, result terminology, units, reference sequences and relevant test statistical performance (such as clinical and analytical) permit the end user to better interpret laboratory results. Half the laboratories reported using standardized nomenclature in their reports. Not all standardized nomenclature is recognizable by the end user; hence, "non-standardized" nomenclature may be added to a report. Two laboratories include both standardized (specifically HUGO) and colloquial names in their reports [39]. Another challenge for reporting is the selection of "positive/negative" vs. "detected/not detected". CLSI MM03 recommends the use of "detected" vs. "not detected" for infectious organisms rather than "positive" or "negative" [40]. For inherited diseases, laboratories may use "present/absent" for specific gene variants and conclude with a description as to whether the genotype is homozygous, heterozygous, composite heterozygous and so on. However, if the assay is cleared or approved by a regulatory agency, then the package insert recommendations for reporting may be followed. Thirty-three percent of laboratories included specific reporting language for assays cleared or approved by a regulatory agency in their reports.

\section{Units and reference sequences}

ISO 15189 recommendations aim to improve standardization results through recommending the inclusion of international system of units (SI), units traceable to SI units or "other appropriate units". This recommendation raises considerable challenges for molecular diagnostics reporting as SI units are not available for many analytes. Prior to the introduction of the international unit (IU), quantification of hepatitis $C$ viral load exemplified the heterogeneity of results for different testing methods. Although all the results were reported in copies per milliliters, the conversion factors of $0.9,2.7,3.4,3.8$ and 5.2 were required to transform copies/mL to IU for each testing methodology [41]. Similarly, the introduction of the International Scale (IS) unit for BCR-ABL1 quantification harmonized reported results across laboratories. Prior to the IS unit's introduction, the Minimal Residual Disease (MRD) proficiency testing survey by the College of American Pathology (CAP) documented numerous reporting units (such as ratio, \% ratio, RNA copies, cell numbers, \% Log BCR-ABL1, copies/mg, log reduction, etc.). Figure 3 presents the various units used by the molecular laboratories (50\%) reporting quantitative test results. Specific reference sequences may provide a control for testing and interpretation of sequence data. For laboratories performing sequencing or multiple/ complex single nucleotide polymorphism (SNP) testing, $69 \%$ specified a reference sequence in their reports and $46 \%$ reported variants of unknown or undetermined significance (VUS). Relevant analytical and clinical performance of the molecular diagnostic assay was not always included in the reports issued by participating laboratories [42]. For laboratories reporting quantitative values, $46 \%$ of laboratories included either the Limit of 


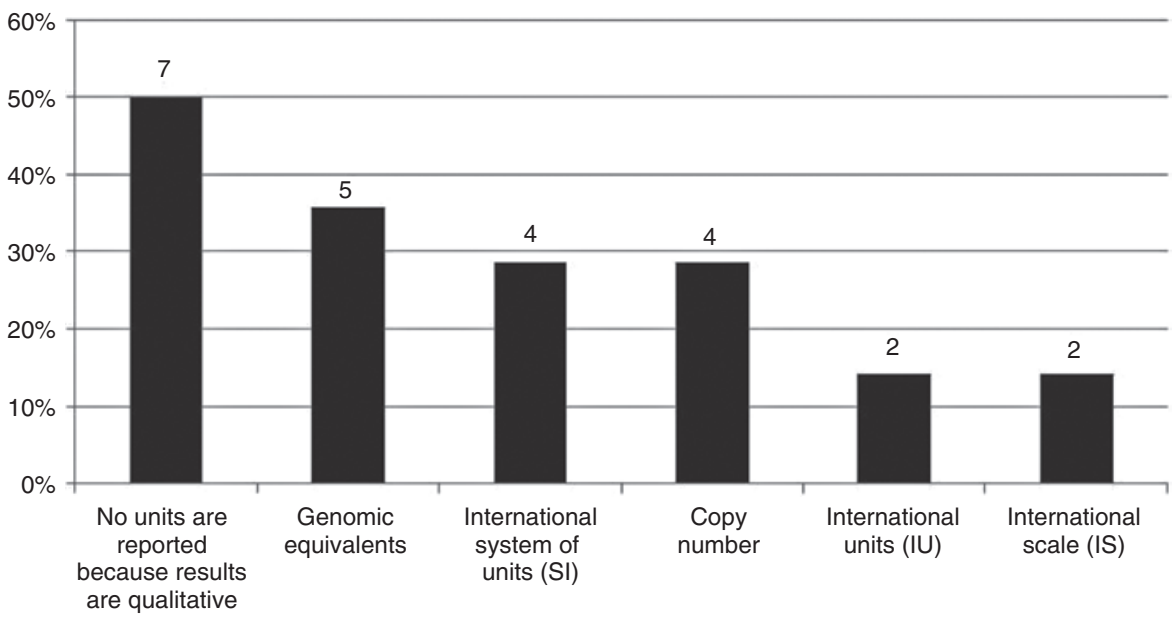

Figure 3: Units of measure for molecular diagnostics.

The number of laboratories reporting specific units of measure is listed above each category. Laboratories not reporting units did not use quantitative tests.

Detection (LOD) and/or the Limit of Quantification (LOQ) in their reports. Measures of uncertainty (for example, $\% \mathrm{CV}, 95 \%$ confidence intervals, etc.) were included in reports by $43 \%$ of surveyed laboratories. The low adherence rates for these recommendations among surveyed laboratories may indicate multiple needs in order to standardize reports.

\section{VUS and secondary findings}

Variants of undetermined/unknown significance (VUS) can be identified with nucleic acid sequencing technologies [43, 44]. Such variants receive this classification when the genotype-phenotype correlation is not known or unclear. The clinical significance of these variants may not be known at the time of diagnosis but may become relevant in the future. A minority of laboratories (46\%) performing sequence analysis reported VUS. This low percentage may reflect the difference between laboratories performing complex genotyping using technologies such as parallel sequencing vs. laboratories offering basic genotyping (such as SNP testing for Factor 5 Leiden) or targeted infectious disease testing. Additional discussion on interpreting and reporting biocurated VUS will be addressed in the interpretation section.

Secondary findings (SF), also known as, incidental findings (IF), may occur if the laboratory performs a test and identifies an organism (or genetic variant) that was not requested $[44,45]$. For instance, in response to a request for Gardnerella vaginalis, a laboratory may erroneously perform Neisseria gonorrhoeae (NG) testing. This secondary finding could have serious implications if the accidental testing identified a medically actionable result. Additionally, the laboratory may perform panels of tests regardless of whether specific analyte(s)/ measurand(s) were requested. The responses for this question were as follows: $29 \%$ reported SF/IF, 21\% did not report SF/IF and $21 \%$ had variable practices depending on the test and test result. One laboratory stated that SF were eliminated when targeted sequencing was performed. Another laboratory identified SF when $B R C A 1$ but not $B R C A 2$ sequencing tests (and vice versa) was ordered. In this scenario, the laboratory routinely performs and reports sequence results from both genes. When a test is accidentally performed due to technical or workflow error and the result could impact patient care, one participant stated that their laboratory would ask the healthcare provider for revised requisition for the specific test that was erroneously performed. After receipt of the revised requisition, the SF/IF was reported. With respect to releasing results from test panels, reporting in toto vs. singly had varied responses among surveyed laboratories. Fifty-four percent of laboratories performing panels reported all results at one time.

\section{Comments on reports}

As outlined by the Vasikaran et al. [46], interpretative comments may facilitate aspects of the information hierarchy by transitioning data to information to knowledge to wisdom (DIKW). The DIKW approach to interpretative comments on reports can aid clinical decision making. For instance, such statements may describe sample quality or specimen acceptance/rejection criteria. These comments provide ongoing education to healthcare providers. Eighty 
Table 4: Examples of specimen comments included in molecular laboratory reports.

Poor specimen quality or quantity

- Low DNA quality, low DNA quantity

- The result was inconclusive due to the lack of sufficient cellularity. Recommend recollection and retesting

- The testing could not be performed due to the specimen not having sufficient quantity (QNS). Recommend recollection and retesting

- ATTENTION: Results could have been compromised by low sample quality. We recommend testing a new sample Wrong specimen type

- Only a peripheral blood is acceptable

Specimen viability compromised

- Hemolysis was observed to this Blood

percent of laboratories provided comments regarding inadequate specimen quality and rejection criteria in their reports. Table 4 contains comments provided by surveyed laboratories. Fifty-three percent of laboratories also stated in their reports which specimen types have been validated. Most laboratories did not accept testing from unvalidated specimens. However, $36 \%$ of laboratories who performed testing on unvalidated samples types provided a comment in the report that the specimen type had been not validated. Laboratories provided such comments in the body (specifically 93\%) of the report vs. the disclaimer section (specifically 7\%). When testing is erroneously performed on two different platforms with discordant results, one laboratory included a comment that a discordant finding was determined using a different platform.

Confusion pertaining to recommendations for so called "critical risk results" occurred in the survey [47]. Critical risk results generally require immediate medical intervention as they indicate a potentially life threatening disease/condition. Defining critical results or values may involve collaboration between the healthcare provider and the laboratory and may be subjective [47]. Some critical values may influence the type and course of treatment. For example, the reporting of the causative agent of meningitis/encephalitis or pneumonia would be a critical risk result. Detection of specific viral and/or bacterial entities in cerebral spinal fluid (CSF) from such patients triggers significantly different treatment modalities. Other examples include the increase in BCR-ABL1 mRNA in chronic myeloid leukemia (CML) patients. Detection of $D P Y D^{*} 2 A / 2 A$ that indicates dihydropyrimidine dehydrogenase deficiency (DPD) as well as genotyping results that predict thiopurine S-methyltransferase (TPMT) deficiency might be considered as critical risk results because these findings would be important for drug dosage adjustment to avoid life-threatening side effects. Without the timely reporting of these analyses, the treatment may be delayed. Although there was confusion regarding this question, $47 \%$ of laboratories stated that they reported critical risk results. Some laboratories designated the critical result in bold font, others had a comment "critical results," whereas others had internal processes to contact the primary healthcare provider and hospital personnel. Twenty-seven percent of laboratories reported that the tests that were offered did not qualify as "critical results".

\section{Interpretation and clinical decision support}

The laboratory may provide interpretation and clinical support in the report. One of the limitations for providing interpretation may be absence or lack of access to relevant clinical information. For this reason, the survey requested participants to comment whether the report stated that the interpretation of the result required clinicopathological correlation. Fifty-three percent of laboratories responded that their report included this language. Laboratories included this statement in the body of the report (50\%), disclaimer (38\%) or website (13\%).

Sixty-seven percent of laboratories included result interpretation in their reports. Seventy percent of laboratories performing tests for multiple single nucleic polymorphisms (SNP) provided interpretation for each SNP along with the interpretation of the entire gene panel in their report. For example, pharmacogenetic testing for multiple SNPs in CYP2D6 may be reported individually and with a final interpretation (such as CYP2D6 Poor Metabolizer). Interpretation may require algorithms. Fifty percent of laboratories using algorithms for interpreting results provide access to the reference either directly in the report, via a link or by providing the algorithm upon request. Specific clinical advice was routinely provided by $40 \%$ of laboratories. One laboratory would provide clinical guidance in limited scenarios such as the presence of an abnormal karyotype. Biological reference intervals, clinical decision points or diagrams supporting clinical decision were provided by $40 \%$ of laboratories; however, many laboratories did not perform quantitative testing making this ISO 15189 recommendation not applicable for some molecular testing. Although not a recommendation by ISO 15189, $60 \%$ of laboratories provided previous molecular findings from the patient in the report. For example, some laboratories include prior molecular results for high risk human papillomavirus (HPV), herpes simplex virus (HSV) and Trypanosoma cruzi. Twenty-six percent of laboratories include results derived as part of a research program with 


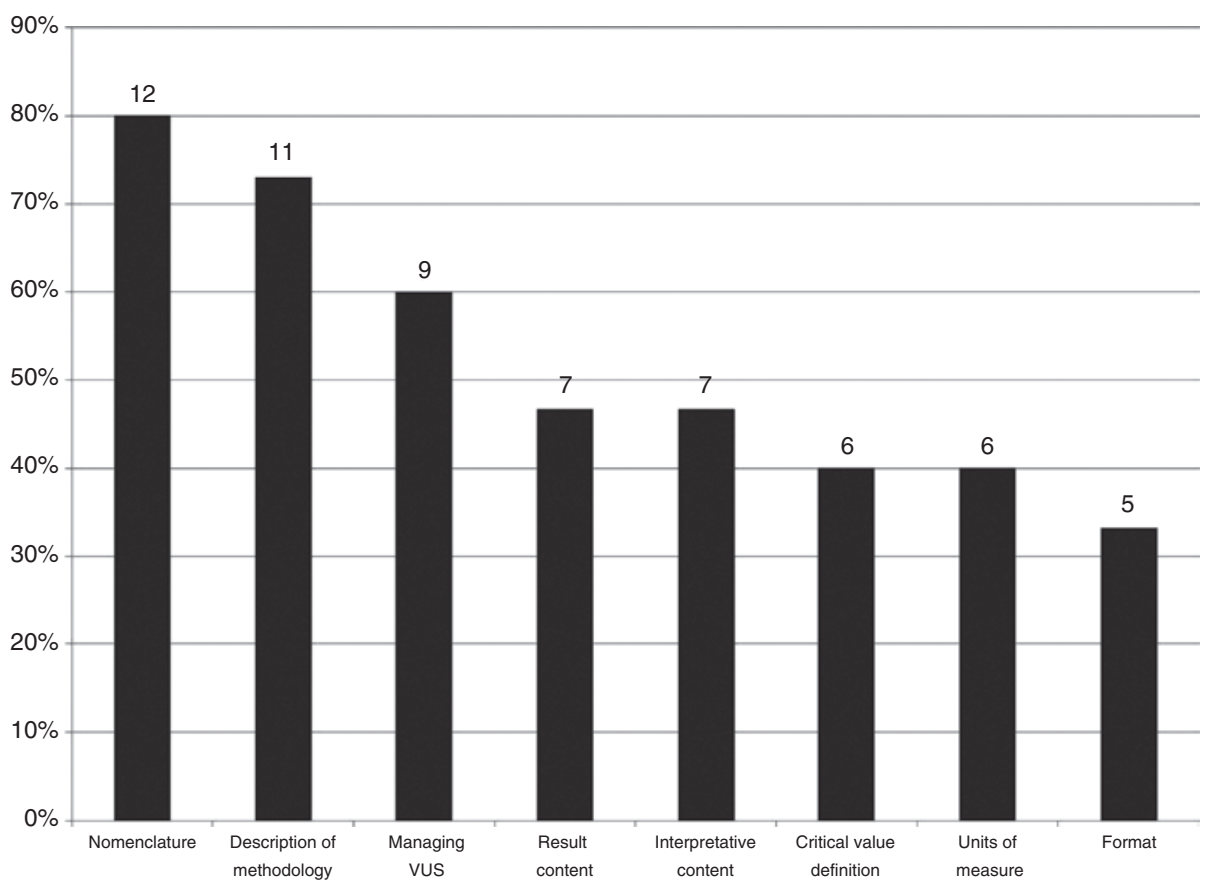

Figure 4: Self reported areas requiring standardization.

The number of laboratories reporting specific areas is listed above each category.

one laboratory stating that the participants were part of a clinical research trial. Seventy-three percent of laboratories also stated the person(s) reviewing the final results as part of the report. Some molecular diagnostic test results (such as some genetic or infectious disease results) may require counseling with the patient. Fifty-seven percent of laboratories report that measures are taken not to provide the information to the patient directly. One such measure includes releasing the information directly to the healthcare provider.

Interpreting and managing variants of undetermined/ unknown significance (VUS) is challenging. One approach is to biocurate the sequence with periodic review. Biocuration with review permits the laboratory to identify and report clinically significant outcomes associated with the variant [48]. Of the laboratories performing biocuration, the review was performed once every 3 months $(n=1)$, once a year $(n=2)$, once every 3 years $(n=1)$ and at different times as clinically indicated for the individual patient. The laboratories would release a revised report if the variant became clinical significant.

\section{Needs assessment}

Survey participants were also asked to select areas or topics they considered needing standardization. Participants were permitted to designate multiple areas or topics.
Nomenclature and description of methodology were most frequently cited by $80 \%(n=12)$ and $72 \%(n=11)$ of survey respondents, respectively (Figure 4). Sixty percent $(n=9)$ of the laboratories stated a need to standardize management of VUS report content with $47 \%(n=7)$ expressing a need for standardization of result and interpretative content. Reporting aspects as divergent as the use of critical values and units of measure were viewed by $40 \%(n=6)$ of laboratories as needing harmonization. Even the least cited category of "format" was indicated as an area needing standardization by $30 \%(n=5)$ of laboratories. All categories generated multiple requests for standardization. In addition, each participant reported at least four or more aspects of the molecular report requiring standardization. This feedback underscores the current heterogeneity of reports and the desire of laboratories to seek standardization in all aspects of molecular diagnostic reports.

\section{Discussion}

Standardization of patient care involving molecular diagnostics occurs when laboratories follow similar guidelines and processes. In this survey, the IFCC C-MD selected recommendations from ISO 15189 pertaining to the reporting phase for the postexamination process of testing and added questions to generate a broader discussion on not yet 
standardized reporting practices. Because the maximum participation rate was 16 and some regions (such as South America) had a single country respond, the outcome may not fully represent the practices in the many molecular testing laboratories and countries around the world. Additional bias may be associated with the selection of participating laboratories as these laboratories are internationally recognized for their experience with performing molecular diagnostic tests. Given these caveats, the surveyed laboratories followed explicit recommendations of ISO 15189, section 5.8 and 5.9.1, at rates that were typically greater than 80\%. However, ISO 15189 also contains numerous recommendations with the phrases "where appropriate", "where applicable", "when relevant to patient care" that may make standardization of reporting practices for molecular results difficult. The survey was extensive and appears to have lost one initial participant during the process. Future surveys will be more concise and will clarify questions with examples. Questions pertaining to "critical risk values" for molecular diagnostics received several comments that underscored the absence in standardization for defining this term. The advent of non-human, web-based links and the use of the reporting language not commonly used in the concerned regions present both opportunities and challenges. Communication advances (i.e. links to websites, etc.) allow access to detailed and most current information for report components, but they also require the healthcare provider and/or patient have access to electronic and software tools. All participants reported at least four categories (additionally, each category was identified multiple times) of the molecular report as requiring standardization. As healthcare providers use and merge multiple molecular diagnostic reports into an electronic health record (EHR), the heterogeneity in report components represents a potential safety issue for patient management as healthcare providers attempt to interpret molecular diagnostic findings. In conclusion, the survey identified several postexamination reporting aspects needing standardization and provides data that will assist professional organizations (such as the IFCC C-MD) to educate and support molecular diagnostic laboratories.

Acknowledgments: The IFCC C-MD would like to acknowledge the guidance and support of the IFCC's Scientific Division, specifically Dr. Philippe Gillery and Dr. Tsutomu Nobori as well as the IFCC staff, specifically Paola Bramati, Patrizia Sirtori and Silvia Cardinale.

Author contributions: All the authors have accepted responsibility for the entire content of this submitted manuscript and approved submission.

Research funding: None declared.
Employment or leadership: None declared.

Honorarium: None declared.

Competing interests: The funding organization(s) played no role in the study design; in the collection, analysis, and interpretation of data; in the writing of the report; or in the decision to submit the report for publication.

\section{References}

1. ISO 15189:2012E 2012. Available from: https://www.iso.org/ standard/56115.html.

2. Payne DA, Baluchova K, Peoc'h KH, van Schaik RH, Chan KC, Maekawa $\mathrm{M}$, et al. Pre-examination factors affecting molecular diagnostic test results and interpretation: a case-based approach. Clin Chim Acta 2017;467:59-69.

3. Lubin IM, Caggana M, Constantin C, Gross SJ, Lyon E, Pagon RA, et al. Ordering molecular genetic tests and reporting results. J Mol Diagn 2008;10:459-68.

4. Gulley ML, Braziel RM, Halling KC, Hsi ED, Kant JA, Nikiforova $M N$, et al. Clinical laboratory reports in molecular pathology. Arch Pathol Lab Med 2007;131:852-63.

5. Claustres M, Kozich V, Dequeker E, Fowler B, Hehir-Kwa JY, Miller K, et al. Recommendations for reporting results of diagnostic genetic testing (biochemical, cytogenetic and molecular genetic). Eur J Hum Genet 2014;22:160-70.

6. Matsuda I, Niikawa N, Sato K, Suzumori K, Fukushima Y, Fujiki N, et al. Guidelines for genetic testing. The Japan Society of Human Genetics, Council Committee of Ethics. J Hum Genet 2001;46:163-5.

7. International Federation for Clinical Chemistry and Laboratory Medicine (IFCC) Committee for Molecular Diagnostics (C-MD). Available from: http://www.ifcc.org/ifcc-scientific-division/sdcommittees/c-md/. Accessed: 20 Apr 2018.

8. Molecular Diagnostic Centres of the International Federation of Clinical Chemistry and Laboratory Medicine. Available from: http://www.ifcc.org/ifcc-scientific-division/sd-committees/c-md/ moleculardiagnosticcentresoftheifcc/. Accessed: 20 Apr 2018.

9. IFCC Molecular Diagnostic Centres. Available from: http://www. ifcc.org/ifcc-scientific-division/sd-committees/c-md/moleculardiagnosticcentresoftheifcc/. Accessed: 20 Apr 2018.

10. SurveyMonkey ${ }^{\circledR}$. Available from: https://www.surveymonkey. com/. Accessed: 12 Sep 2017.

11. College of American Pathologists (CAP). Available from: http:// www.cap.org. Accessed: 20 Apr 2018.

12. CDC links to Clinical Laboratory Improvement Amendments (CLIA). Available from: https://wwwn.cdc.gov/clia. Accessed: 20 Apr 2018.

13. Clinical Laboratory Improvement Amendments (CLIA). Standards and certifications: laboratory requirements (42 CFR 493) Electronic Code of Federal Regulations e-CFR. Available from: https://www.ecfr.gov/cgi-bin/text-idx?SID=1248e3189da5e5f9 36e 55315402 bc 38 b\&node $=p t 42.5 .493 \&$ rgn $=$ div5 . Accessed: 20 Apr 2018.

14. Comité Français d'Accréditation (COFRAC). Available from: https://www.cofrac.fr. Accessed: 20 Apr 2018.

15. Deutsche Akkreditierungsstelle (DAkkS). Available from: https:// www.dakks.de. Accessed: 20 Apr 2018. 
16. European Accreditation (EA). Available from: http://www. european-accreditation.org/. Accessed: 10 Nov 2017.

17. The Health Reference Laboratory, Ministry of Health of IRAN. Available from: http://irandataportal.syr.edu/ministry-ofhealth-and-medical-education. Accessed: $20 \mathrm{Apr} 2018$.

18. International Accreditation Forum (IAF). Available from: http:// www.iaf.nu/. Accessed: 20 Apr 2018.

19. International Laboratory Accreditation Cooperation (ILAC). Available from: http://ilac.org/. Accessed: 20 Apr 2018.

20. International Organization of Standardization (ISO). Available from: https://www.iso.org. Accessed: 20 Apr 2018.

21. National Accreditation Board of Testing and Calibration Laboratories (NABL). Available from: http://www.nabl-india.org. Accessed: 20 Apr 2018.

22. National Pathology Accreditation Advisory Council (NPAAC) via National Association of Testing Authorities, Australia (NATA). Available from: http://www.health.gov.au/npaac. Accessed: 20 Apr 2018.

23. Netherlands Institute for Accreditation (NIAZ). Available from: https://www.niaz.nl/english. Accessed: 20 Apr 2018.

24. Slovak National Accrediation Service (SNAS). Available from: http://www.snas.sk/. Accessed: 20 Apr 2018.

25. American College of Medical Genetics and Genomics (ACMG). Available from: https://www.acmg.net. Accessed: 20 Apr 2018.

26. Association of Clinical Genetic Science (ASHG). Available from: http://www.acgs.uk.com. Accessed: 20 Apr 2018.

27. Association for Molecular Pathology (AMP). Available from: https://www.amp.org/. Accessed: 20 Apr 2018.

28. Australian Government Department of Health Therapeutic Goods Administration. Available from: https://www.tga.gov.au. Accessed: 20 Apr 2018.

29. Canadian Standards Associations (CSA). Available from: www. csagroup.org. Accessed: 20 Apr 2018.

30. Clinical Laboratory Standardization Institute (CLSI). Available from: https://clsi.org/. Accessed: 10 Nov 2017.

31. Genetic Services Quality Committee of the European Society of Human Genetics (ESHG). Available from: https://www.eshg.org/ home.0.html. Accessed: 10 Nov 2017.

32. Japanese Committee for Clinical Laboratory Standards (JCCLS). Available from: http://jccls.org/english/index.html. Accessed: 20 Apr 2018.

33. Israel Health Ministry Standardization. Available from: https:// www.health.gov.il. Accessed: 20 Apr 2018.

34. The Organisation for Economic Co-operation and Development (OECD). Available from: http://www.oecd.org/sti/biotech/oecdguidelinesforqualityassuranceingenetictesting.htm. Accessed: 10 Nov 2017.

35. Richtlinie der Bundesärztekammer; German Chamber of Physicians. Available from: http://www.bundesaerztekammer.de. Accessed: 10 Nov 2017.
36. Swiss Society of Medical Genetics (SSMG). Available from: http://sgmg.ch/?page_id=5. Accessed: 20 Apr 2018.

37. International Classification of Diseases [ICD-10]. Available from: http://www.who.int/classifications/icd/en/. Accessed: 12 Sep 2017.

38. Online Mendelian Inheritance in Man (OMIM). Available from: https://www.omim.org/. Accessed: 12 Sep 2017.

39. Kalman LV, Agundez J, Appell ML, Black JL, Bell GC, Boukouvala $S$, et al. Pharmacogenetic allele nomenclature: international workgroup recommendations for test result reporting. Clin Pharmacol Ther 2016;99:172-85.

40. Day SP, Jackson CL, Nolte FS, Tezak-Fragale Z, Clinical and Laboratory Standards Institute (CLSI). Molecular diagnostic methods for infectious diseases. 3rd ed. CLSI report MMO3. Wayne, PA: Clinical and Laboratory Standards Institute, 2015.

41. Pawlotsky JM. Use and interpretation of virological tests for hepatitis C. Hepatology 2002;36(5 Suppl 1):S65-73.

42. Rousseau F, Lindsay C, Charland M, Labelle Y, Bergeron J, Blancquaert I, et al. Development and description of GETT: a genetic testing evidence tracking tool. Clin Chem Lab Med 2010;48:1397-407.

43. Vears DF, Senecal K, Borry P. Reporting practices for variants of uncertain significance from next generation sequencing technologies. Eur J Med Genet 2017;60:553-8.

44. Hehir-Kwa JY, Claustres M, Hastings RJ, van Ravenswaaij-Arts C, Christenhusz G, Genuardi M, et al. Towards a European consensus for reporting incidental findings during clinical NGS testing. Eur J Hum Genet 2015;23:1601-6.

45. Kalia SS, Adelman K, Bale SJ, Chung WK, Eng C, Evans JP, et al. Recommendations for reporting of secondary findings in clinical exome and genome sequencing, 2016 update (ACMG SF v2.0): a policy statement of the American College of Medical Genetics and Genomics. Genet Med 2017;19:249-55.

46. Vasikaran S, Sikaris K, Kilpatrick E, French J, Badrick T, Osypiw J, et al. Assuring the quality of interpretative comments in clinical chemistry. Clin Chem Lab Med 2016;54:1901-11.

47. Young AN, Dighe A, Beastall G, Contis LC, Fernandez-Calle P, Gryko CM, et al., Clinical and Laboratory Standards Institute (CLSI). Management of critical and significant-risk results. 1st ed. CLSI guideline G47ES. Wayne, PA: Clinical and Laboratory Standards Institute, 2015.

48. Strande NT, Riggs ER, Buchanan AH, Ceyhan-Birsoy O, DiStefano M, Dwight SS, et al. Evaluating the clinical validity of gene-disease associations: an evidence-based framework developed by the clinical genome resource. Am J Hum Genet 2017;100:895-906.

Supplementary Material: The online version of this article offers supplementary material (https://doi.org/10.1515/cclm-2017-1080). 